\title{
SIMPLE MODELS AND METHODS FOR ESTIMATING THE ULTRASONIC REFLECTIVITY OF SPOT WELDS
}

William B. Davis

Lawrence Berkeley National Laboratory, MS 46A-1123B, One Cyclotron Road, Berkeley CA 94720.

\begin{abstract}
This paper describes models and methods for estimating the acoustic reflectivity of the welded interfaces between spot-welded sheets from normal-incidence pulse-echo ultrasound signals. The simple geometry of the problem allows an abstraction that does not resort to complex wave equations. Instead, a reflectivity model predicts the timing and amplitude of the echoes arriving at the probe. This reflectivity model is nested in a signal processing model; recovering reflectivity first requires deconvolution to recover discrete impulses from the probe signal, then processing these with the reflectivity model. Reflectivity maps of spot welds generated with this model show promise for predicting weld quality.
\end{abstract}

\section{INTRODUCTION AND OVERVIEW}

Acoustic reflectivity $R$ is defined as the unitless share of acoustic energy incident on a spot-welded interface that is reflected. Also transmissivity $T$ is the remaining share of signal energy that is transmitted across the interface. It is assumed that $R+T=1$. To estimate reflectivity, a model is described, and fitting techniques applied.

The model and the fitting techniques are described in two stages. A reflectivity model is embedded in a signal processing model. Using the signal processing model, deconvolution is first applied to the raw signal to recover the discrete impulses. Then using the reflectivity model, NLLS is applied to the discrete impulses to recover an estimate of the reflectivity.

The reflectivity model has two forms, corresponding to equal and unequal thickness sheets. The simplest form of the model, for two sheets of equal thickness, has proven successful for discriminating weld quality, and a fast implementation has been developed. The unequal-thickness sheets model has also been verified empirically, but the implementation is still slow.

Both forms of the model have been developed for two- and three-sheet stackups. Only the two-sheet models have been tested empirically. The three-sheet models present additional complexity and measurement problems, and have not yet been validated. The success of the two-sheet models, however, suggests that the three-sheet approach is correct in theory.

The following sections follow the same development as this introduction: first the signal processing model and deconvolution method are described; then the equal thickness sheets reflectivity model, and a Gauss-Newton implementation of NLLS to recover an estimate of reflectivity, is introduced; the section following describes the unequal thickness sheets model; and, the final section introduces the extension to three sheets. 


\section{SIGNAL PROCESSING MODEL AND DECONVOLUTION}

Standard linear systems theory describes a signal $S(t)$ as a convolution $\otimes$

$$
S(t)=H(t) \otimes \sum_{i=1}^{I} \beta_{i} \delta\left(t-\tau_{i}\right)
$$

of a transducer function $H(t)$ and an impulse train $\left(\beta_{i}, \tau_{i}\right)$, where $\beta_{i}$ is the amplitude and $\tau_{i}$ the time of arrival of impulse $i$, and $\delta$ is the dirac function. The transducer function can be assumed to be a Gaussian wavelet

$$
H(t)=\beta \exp \left[-\alpha(t-\tau)^{2}\right] \cos [2 \pi f(t-\tau)+\phi]
$$

This formulation is useful for simulation. Demirli and Saniie provide the formulae for the Gauss-Newton method in an application of nonlinear least-squares (NLLS) to recover the parameters of the above family of Gaussian wavelets [DS01a\&b].

Recovering the impulse train when $H(t)$ is unknown is a signal processing task known as blind deconvolution [Hay00a\&b, CA02]. The following approach does not require specification of $H(t)$, and requires only the sheet thicknesses and ultrasound velocity as input. First calculate the sequence of expected impulse arrival times $\mathrm{E}\left[\tau_{i}\right]$ for the interface and back surfaces, as sheet thicknesses times the speed of the ultrasound in the medium. Then with this sequence of expected arrival times, for each impulse $i=1$ to $I$,

$$
\beta_{i}=\max \left|S\left(\mathrm{E}\left[\tau_{i}\right]-\left\lceil\frac{\Delta \mathrm{E}\left[\tau_{i}\right]}{2}\right\rceil+1, \ldots, \mathrm{E}\left[\tau_{i}\right]+\left\lceil\frac{\Delta \mathrm{E}\left[\tau_{i+1}\right]}{2}\right\rceil-1\right)\right|,
$$

the amplitude $\beta_{i}$ is a local maximum of the signal magnitude $|\mathrm{S}(\tau)|$, in the vicinity of the expected impulse arrival time. With this algorithm for blind deconvolution, further signal processing requires only the discrete impulse train. All further discussion therefore concerns only the timing and amplitude of discrete impulses received by the probe.

\section{EQUAL-THICKNESS SHEETS}

\section{Reflectivity Model}

To develop a model of acoustic energy transmission through a spot weld joining two equal-thickness sheets, first consider a single sheet. When an ultrasonic plane wave is induced on one surface, it reflects off the opposite or back surface, then again off the front surface, etc., until the signal is dissipated by material attenuation. The period of the echoes is the time it takes the signal to traverse twice the thickness of the sheet. The amplitude of the initial impulse decays exponentially over time at a constant, material- and frequencydependent rate $d,|S(t)|=|S(0)| e^{-d t}$. 

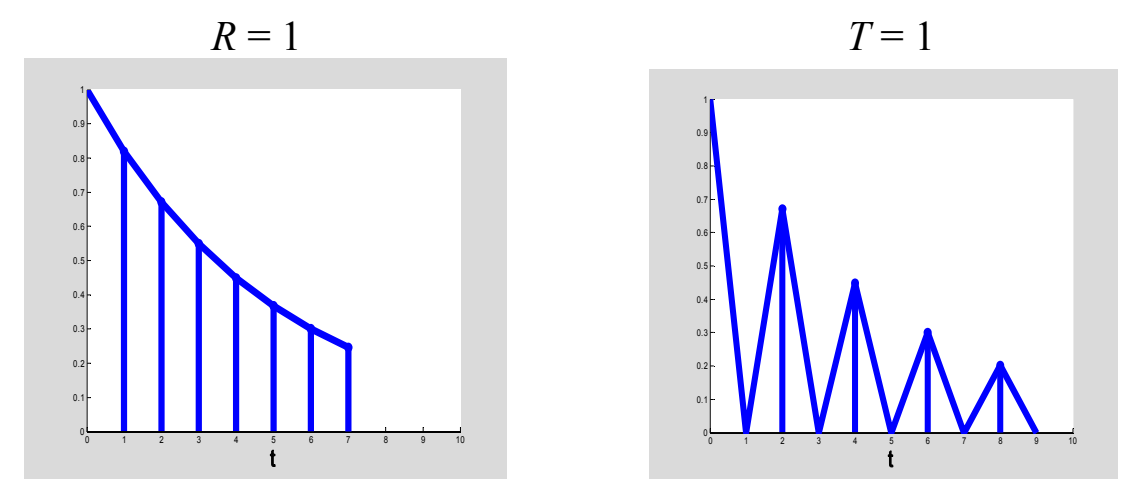

FIGURE 1. Discrete impulse trains for $R=1, T=1$.

Now consider transmission of acoustic energy through two sheets of equal thickness. With perfect transmission $(T=1)$, the echo period is twice the previous, the time it takes the signal to traverse the thickness of four sheets. With perfect reflection $(R=1)$, the signal would revert to the one-sheet model. These cases are illustrated in Figure 1. In practice, however, some share $T$ of the signal is transmitted through the weld, and the remainder $R=$ $1-T$ is reflected $(T, R \in[0,1])$. Describing these intermediate cases is the task of the reflectivity model.

The reflectivity model is derived from a pair of difference equations that describe the amplified signal received at each surface as proportional to the signal reflected by the weld from that surface plus the signal transmitted through the weld from the opposite surface, as follows:

$$
\begin{aligned}
& \beta_{t+1} e^{d}=R \beta_{t}+T \partial_{t} ; \\
& \partial_{t+1} e^{d}=T \beta_{t}+R \partial_{t} .
\end{aligned}
$$

It can be verified that the front-surface state equation for this system is

$$
\beta_{t}=\beta_{0} e^{-d t} \sum_{k=0}^{\lfloor t / 2\rfloor}\left(\begin{array}{c}
t \\
2 k
\end{array}\right) R^{t-2 k} T^{2 k}
$$

This equation sums the contributions to each impulse of all possible sequences of interface transmissions and reflections. It predicts the impulse train given the values of the parameters $R, \beta_{0}$, over $t=1$ to $T$. Normalizing by the initial impulse $* \beta_{0}{ }^{-1}$, and amplifying by the material attenuation rate $* \mathrm{e}^{d t}$, the following Figure 2 charts (normalized, amplified) impulse trains for several intermediate values of $R=\beta_{1}$. 


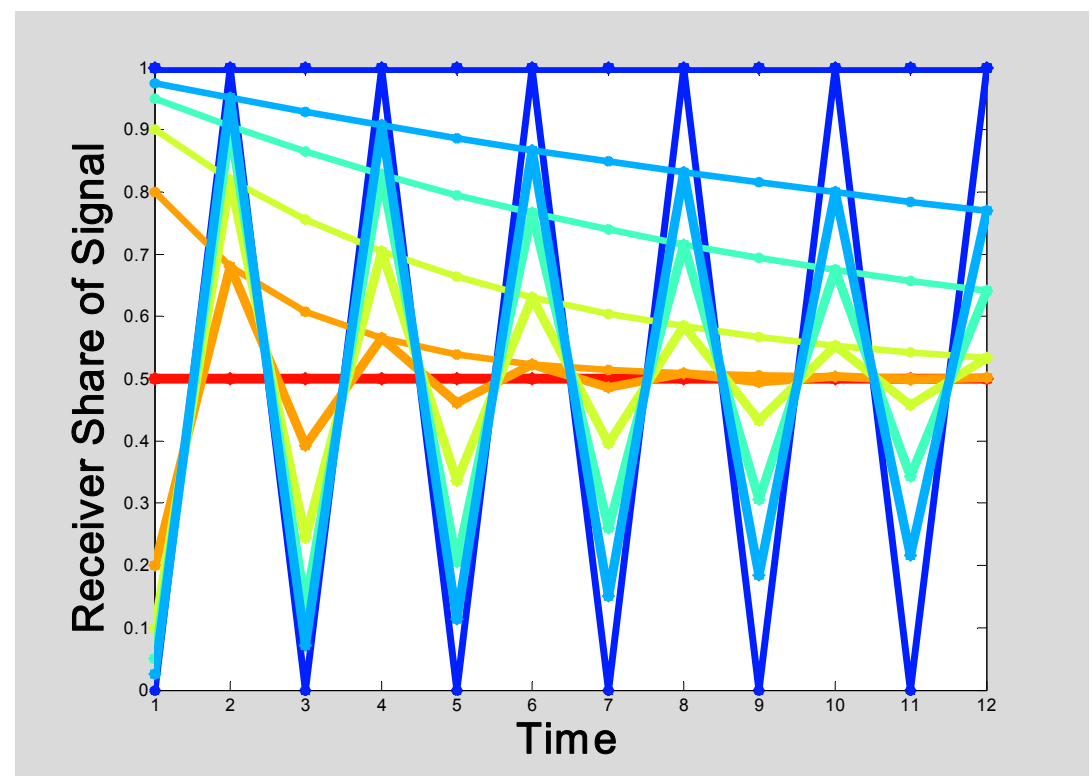

FIGURE 2. Normalized, amplified impulse trains for a range of reflectivities, $R=\beta_{1}$.

This model predicts an equilibrium share of $\beta_{\infty}=0.5$ for all interior values of $R$. That 0.5 is an equilibrium can be demonstrated by noting that if $\beta_{\mathrm{t}}=0.5$, then $\beta_{\mathrm{t}}=\partial_{\mathrm{t}}$, and

$$
\beta_{t+1}=R \beta_{t}+T \partial_{t}=(R+T) \beta_{t}=\beta_{t}
$$

This is consistent with the Second Law of Thermodynamics. Uniform shares is the maximum entropy state, and the entropy of $R$ is the same as the entropy of $1-R$, so entropy is always increasing.

The dynamics of the impulse trains for intermediate values of $R$ are binned into two classes, with the separator at the equilibrium $R^{*}=0.5$. For values of $R>0.5$, there is smooth decay of the share of energy at the receiver, toward the equilibrium share of 0.5 ; for $R<0.5$ there is damped oscillation. In both cases, this damping is independent of and in addition to the exponential damping of the signal due to material attenuation. Furthermore, this damping is the same for reflectivity $R$ as for $1-R$, with the back-surface echoes coinciding. The interface partitions the signal energy between the two sheets, and the closer the transmissivity is to the reflectivity, the quicker the maximum entropy equilibrium uniform shares of 0.5 are realized.

The Gauss-Newton method for nonlinear least-squares can be applied to recover an estimate of the reflectivity parameters $\boldsymbol{\theta}=\left(R, \beta_{0}\right)$. Because the initial impulse is usually set to saturate the receiver, for maximum information gain at later time periods, the normalization constant $\beta_{0}$ must also be estimated simultaneously. This constant captures everything that contributes to the overall magnitude of the signal, including gain, coupling, and gradual probe element failure. The Gauss-Newton method for NLLS repeatedly updates the parameter vector until convergence as follows. While $\boldsymbol{\theta}^{k}-\boldsymbol{\theta}^{k-1}>\varepsilon$,

$$
\boldsymbol{\theta}^{k+1}=\boldsymbol{\theta}^{k}-\left[\boldsymbol{J}^{\prime}\left(\boldsymbol{\theta}^{k}\right) \boldsymbol{J}\left(\boldsymbol{\theta}^{k}\right)\right]^{-1} \boldsymbol{J}^{\prime}\left(\boldsymbol{\theta}^{k}\right) \boldsymbol{e},
$$


with the Jacobian

$$
\boldsymbol{J}^{T}(\boldsymbol{\theta}) \equiv \frac{\partial \beta(\boldsymbol{\theta} ; t)}{\partial \boldsymbol{\theta}}=\left[\frac{\partial \beta(\boldsymbol{\theta} ; t)}{\partial R} \quad \frac{\partial \beta(\boldsymbol{\theta} ; t)}{\partial S}\right]
$$

having elements

$$
\frac{\partial \beta(t)}{\partial R}=-\frac{\sum_{k=0}^{\lfloor t / 2\rfloor}\left(\begin{array}{c}
t \\
2 k
\end{array}\right)\left[(t-2 k) R^{t-2 k-1} T^{2 k}+2 k R^{t-2 k} T^{2 k-1}\right]}{\sum_{k=0}^{\lfloor t / 2\rfloor}\left(\begin{array}{c}
t \\
2 k
\end{array}\right) R^{t-2 k} T^{2 k}} \text {, and } \frac{\partial \beta(t)}{\partial S}=-\frac{1}{S} .
$$

\section{Experimental Results}

The model for equal-thickness sheets was tested on 36 galvanized mild steel coupons of 1.5 to $1.5 \mathrm{~mm}$. Typical reflectivity colormaps of the welds are provided in Figure 3 .
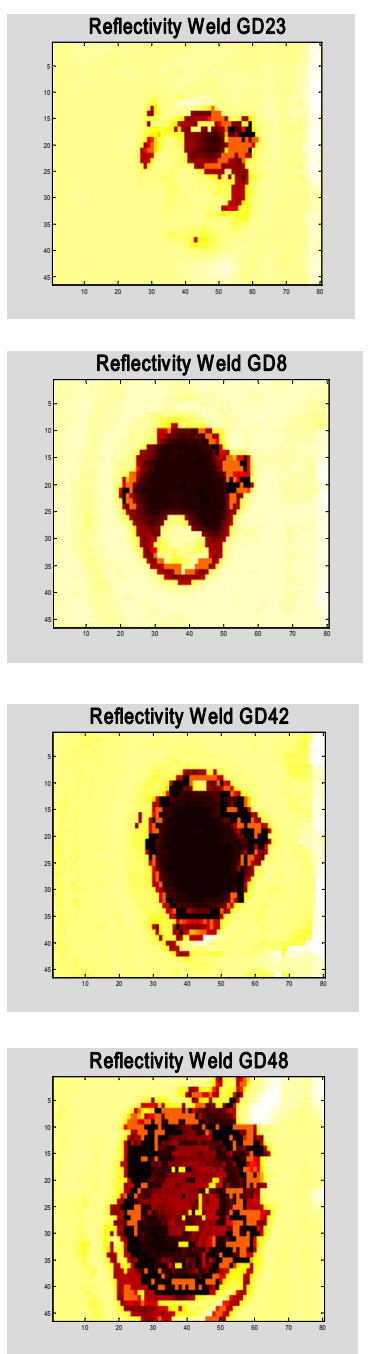
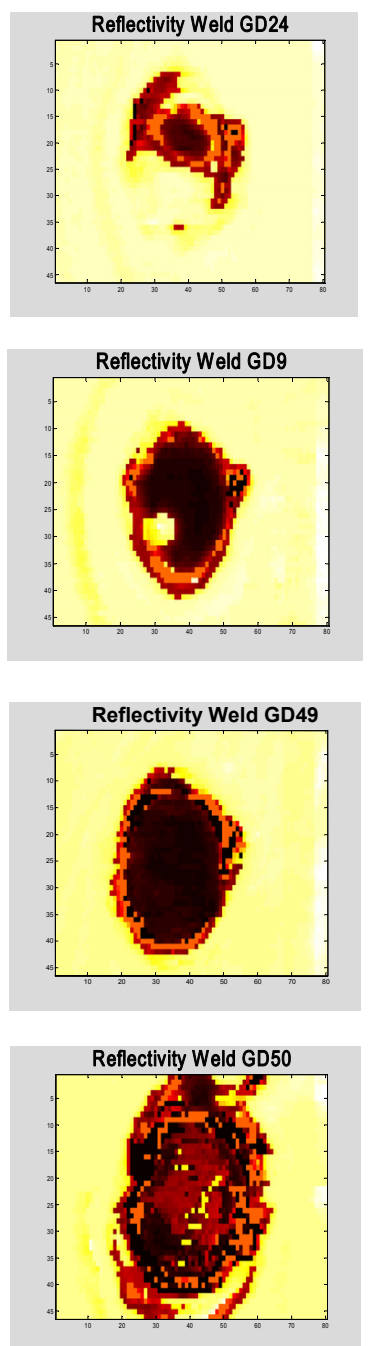
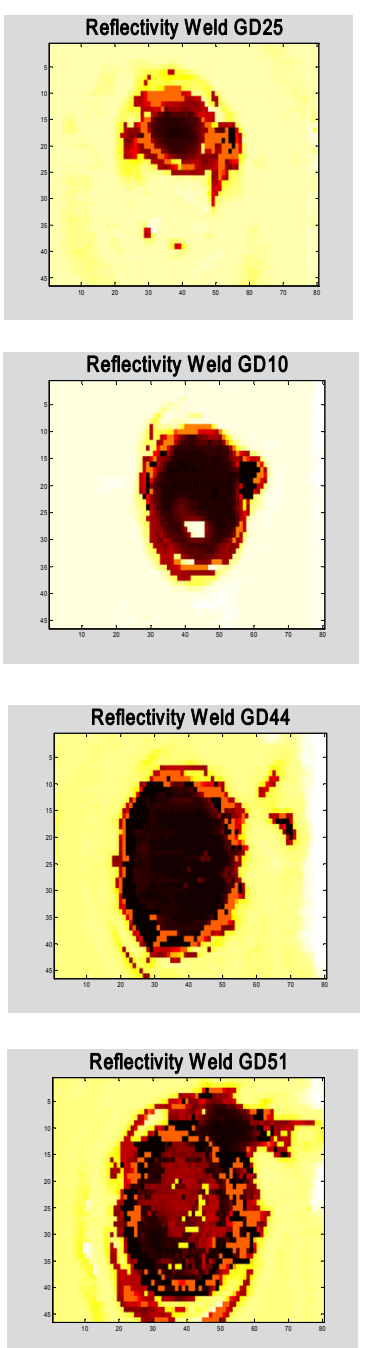
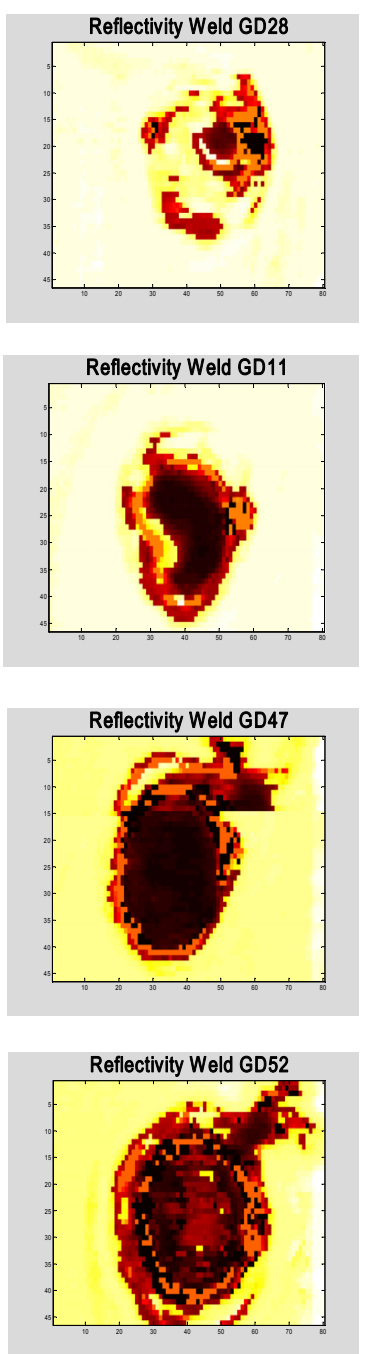

FIGURE 3. Typical reflectivity maps for stick/weak welds (top row), undersized welds (second row), good welds (third row), and burnt welds (bottom row). 
To construct the test coupons, welding current, pressure, and hold time were varied to achieve four target weld qualities: weak/stick, undersized, good, and burnt. The welds were probed with a $10 \mathrm{MHz}$ R/D Tech ultrasonic linear phased array, then the weld qualities confirmed by destructive teardown. Figure 3 depicts reflectivity maps typical of each weld quality.

Of the 36 welds in the sample, only two could not be correctly classified from the reflectivity maps. These two were weak or undersized welds that appeared good because of acoustic transmission across the interface. In both cases, however, these classification errors could be corrected by examining the indentation map (also derived from the probe signals, from the time-of-arrival of the first front-surface echo). Good welds were always observed to have a deeper front-surface indentation, while the indentations for weak and undersized welds were negligible. Therefore, by using both feature maps, $100 \%$ correct classification could be achieved.

\section{UNEQUAL THICKNESS SHEETS}

\section{Reflectivity Model}

For the unequal thickness sheets, or when the front sheet is thin or the indentation relatively deep, the input thickness must be reduced by an orientation-adjusted indentation estimate, recovered from the signal by the time of arrival of the first front-surface echo. Then the following pair of difference equations describes the time of arrival and amplitudes of the impulse train received by the probe:

$$
\begin{gathered}
\beta_{t+2 r / v}=e^{-a 2 r / v} R \beta_{t}+e^{-d(r+s) / v} T \delta_{t+(r-s) / v} \\
\delta_{t+2 r / v}=e^{-d(r+s) / v} T \beta_{t+(r-s) / v}+e^{-d 2 s / v} R \delta_{t+2(r-s) / v}
\end{gathered}
$$

This system of difference equations still describes the arrival times and amplitudes of the back-surface and interface echoes at the probe. The thicknesses of the sheets are $r$ and $s$, so the ratios in the indices and exponentials are time terms (distance/velocity $v$ ). In each period the deconvolved probe signal is still a sum of reflected interface and transmitted back-surface echoes, now appropriately lagged and attenuated.

\section{THREE SHEETS}

\section{Models}

The two sheet model can be extended to simulate the probe response for a three-sheet stackup, with two interfaces and reflectivity parameters $R_{1}$ and $R_{2}$ (and $T_{i}=1-R_{i}$ ). The difference equations that describe the evolution of energy shares between three sheets are:

$$
\begin{gathered}
\beta_{t+1} e^{d}=R_{1} \beta_{t}+T_{1} \delta_{t} \\
\delta_{t+1} e^{d}=R_{2}\left(T_{1} \beta_{t}+R_{1} \delta_{t}\right)+T_{2} \varepsilon_{t} \\
\varepsilon_{t+1} e^{d}=T_{2}\left(T_{1} \beta_{t}+R_{1} \delta_{t}\right)+R_{2} \varepsilon_{t}
\end{gathered}
$$


This system reverts to the two-sheet model if $R_{2}=1$. With initial conditions $\beta_{0}=\beta$ and $\delta_{0}=\varepsilon_{0}=0$, these equations completely specify the probe state $\beta_{t} \forall t$. This system also is consistent with the First and Second Laws of Thermodynamics, because

$$
\beta_{t}+\delta_{t}+\varepsilon_{t}=1 \Rightarrow\left(\beta_{t+1}+\delta_{t+1}+\varepsilon_{t+1}\right) e^{a}=1,
$$

and

$$
\beta_{t}=\delta_{t}=\varepsilon_{t}=1 / 3 \Rightarrow \beta_{t+1} e^{a}=\delta_{t+1} e^{a}=\varepsilon_{t+1} e^{a}=1 / 3 .
$$

Energy is conserved, and uniform shares is the maximum entropy equilibrium to which the model monotonically converges for all interior starting values.

The extension to unequal thicknesses $p, q, r$ is

$$
\begin{gathered}
\beta_{t+2 p / v}=e^{-d p / v} R_{1} \beta_{t}+e^{-d(p+q) / v} T_{1} \delta_{t+2(p-q) / v} \\
\delta_{t+2 p / v}=e^{-d 2 q / v} R_{2}\left(e^{-d p / v} T_{1} \beta_{t}+e^{-d(p+q) / v} R_{1} \delta_{t+2(p-q) / v}\right)+e^{-d r / v} T_{2} \varepsilon_{t+2(p-r) / v} \\
\varepsilon_{t+2 p / v}=e^{-d 2 q / v} T_{2}\left(e^{-d p / v} T_{1} \beta_{t}+e^{-d(p+q) / v} R_{1} \delta_{t+2(p-q) / v}\right)+e^{-d r / v} R_{2} \varepsilon_{t+2(p-r) / v}
\end{gathered}
$$

The three-sheet models have not yet been empirically verified, and are certain to prove more difficult to estimate quickly and accurately. They are, however, still useful for simulation.

\section{ACKNOWLEDGEMENTS}

This work was supported by the Department of Energy under Contract No. DE-ACO376SF00098, and performed at Lawrence Berkeley National Laboratory Engineering. The author thanks Dr. Deb Hopkins and Dr. Fred Reverdy for providing the experimental data, and acknowledges the contributions of USCAR's NDE of Welded Metals Industry Steering Committee.

\section{REFERENCES}

[CA02] A Cichoki, S Amari, 2002. Adaptive Blind Signal and Image Processing (New York: Wiley-Interscience).

[DS01a] R Demirli, J Sanilie, 2001. "Model-Based Estimation of Ultrasonic Echoes: Analysis and Algorithms," IEEE Transactions on Ultrasonics, Ferroelectrics, and Frequency Control, vol. 48 no. 3, pp. 787-802.

[DS01b] R Demirli, J Sanilie, 2001. "Model-Based Estimation of Ultrasonic Echoes: Nondestructive Evaluation Applications," IEEE Transactions on Ultrasonics, Ferroelectrics, and Frequency Control, vol. 48 no. 3, pp. 803-811. 
[Hay00a] Haykin, S, Ed., 2000. Unsupervised Adaptive Filtering: Blind Source Separation (New York: Wiley-Interscience).

[Hay00b] Haykin, S, Ed., 2000. Unsupervised Adaptive Filtering: Blind Deconvolution (New York: Wiley-Interscience).

Haykin, S, 1996. Adaptive Filter Theory (New York: Prentice Hall). 